\title{
Hermite-Hadamard-Fejér type inequalities for harmonically convex functions via fractional integrals
}

\author{
Imdat Iscan ${ }^{1}$, Mehmet Kunt ${ }^{2}$ and Nazli Yazici ${ }^{3}$ \\ ${ }^{1}$ Department of Mathematics, Faculty of Sciences and Arts, Giresun University, 28200, Giresun, Turkey \\ ${ }^{2,3}$ Department of Mathematics, Faculty of Sciences, Karadeniz Technical University, 61080, Trabzon, Turkey
}

Received: 3 June 2016, Accepted: 21 July 2016

Published online: 13 August 2016.

\begin{abstract}
In this paper, firstly, Hermite-Hadamard-Fejér type inequality for harmonically convex functions in fractional integral forms have been established. Secondly, an integral identity and some Hermite-Hadamard-Fejér type integral inequalities for harmonically convex functions in fractional integral forms have been obtained. The some results presented here would provide extensions of those given in earlier works.
\end{abstract}

Keywords: Hermite-Hadamard inequality, Hermite-Hadamard-Fejér inequality, Riemann-Liouville fractional integral, harmonically convex function.

\section{Introduction}

Let $f: I \subseteq \mathbb{R} \rightarrow \mathbb{R}$ be a convex function defined on the interval $I$ of real numbers and $a, b \in I$ with $a<b$. The inequality

$$
f\left(\frac{a+b}{2}\right) \leq \frac{1}{b-a} \int_{a}^{b} f(x) d x \leq \frac{f(a)+f(b)}{2}
$$

is well known in the literature as Hermite-Hadamard's inequality [5].

The most well-known inequalities related to the integral mean of a convex function $f$ are the Hermite Hadamard inequalities or its weighted versions, the so-called Hermite-Hadamard-Fejér inequalities.

In [4], Fejér established the following Fejér inequality which is the weighted generalization of Hermite-Hadamard inequality (1).

Theorem 1. Let $f:[a, b] \rightarrow \mathbb{R}$ be convex function. Then, the inequality

$$
f\left(\frac{a+b}{2}\right) \int_{a}^{b} g(x) d x \leq \int_{a}^{b} f(x) g(x) d x \leq \frac{f(a)+f(b)}{2} \int_{a}^{b} g(x) d x
$$

holds, where $g:[a, b] \rightarrow \mathbb{R}$ is nonnegative, integrable and symmetric to $(a+b) / 2$.

For some results which generalize, improve and extend inequalities (1) and (2) see [1,6,7,15,17].

We recall the following inequality and special functions which are known as Beta and hypergeometric function 
respectively

$$
\begin{gathered}
\beta(x, y)=\frac{\Gamma(x) \Gamma(y)}{\Gamma(x+y)}=\int_{0}^{1} t^{x-1}(1-t)^{y-1} d t, x, y>0 \\
{ }_{2} F_{1}(a, b ; c ; z)=\frac{1}{\beta(b, c-b)} \int_{0}^{1} t^{b-1}(1-t)^{c-b-1}(1-z t)^{-a} d t, c>b>0,|z|<1 \text { (see [12]). }
\end{gathered}
$$

Lemma 1. For $0<\alpha \leq 1$ and $0 \leq a<b$ we have

$$
\left|a^{\alpha}-b^{\alpha}\right| \leq(b-a)^{\alpha} .
$$

$($ see $[14,19])$.

We will now give definitions of the right-hand side and left-hand side Riemann-Liouville fractional integrals which are used throughout this paper.

Definition 1. Let $f \in L[a, b]$. The right-hand side and left-hand side Riemann-Liouville fractional integrals $J_{a+}^{\alpha} f$ and $J_{b-}^{\alpha} f$ of order $\alpha>0$ with $b>a \geq 0$ are defined by

$$
\begin{aligned}
& J_{a+}^{\alpha} f(x)=\frac{1}{\Gamma(\alpha)} \int_{a}^{x}(x-t)^{\alpha-1} f(t) d t, x>a \text { and } \\
& J_{b-}^{\alpha} f(x)=\frac{1}{\Gamma(\alpha)} \int_{x}^{b}(t-x)^{\alpha-1} f(t) d t, x<b,
\end{aligned}
$$

respectively, where $\Gamma(\alpha)$ is the Gamma function defined by $\Gamma(\alpha)=\int_{0}^{\infty} e^{-t} t^{\alpha-1} d t$ (see [12]).

Because of the wide application of Hermite-Hadamard type inequalities and fractional integrals, many researchers extend their studies to Hermite-Hadamard type inequalities involving fractional integrals not limited to integer integrals. Recently, more and more Hermite-Hadamard inequalities involving fractional integrals have been obtained for different classes of functions; see $[3,8,9,16,18,19]$.

In [11], İşcan gave definition of harmonically convex functions and established following Hermite-Hadamard type inequality for harmonically convex functions as follows.

Definition 2. Let $I \subset \mathbb{R} \backslash\{0\}$ be a real interval. A function $f: I \rightarrow \mathbb{R}$ is said to be harmonically convex, if

$$
f\left(\frac{x y}{t x+(1-t) y}\right) \leq t f(y)+(1-t) f(x)
$$

for all $x, y \in I$ and $t \in[0,1]$. If the inequality in (3) is reversed, then $f$ is said to be harmonically concave.

Theorem 2. Let $f: I \subset \mathbb{R} \backslash\{0\} \rightarrow \mathbb{R}$ be a harmonically convex function and $a, b \in I$ with $a<b$. If $f \in L[a, b]$ then the following inequalities holds:

$$
f\left(\frac{2 a b}{a+b}\right) \leq \frac{a b}{b-a} \int_{a}^{b} \frac{f(x)}{x^{2}} d x \leq \frac{f(a)+f(b)}{2} .
$$

(see [11]).

In [10], İşcan and Wu presented Hermite-Hadamard's inequalities for harmonically convex functions in fractional integral forms as follows. 
Theorem 3. Let $f: I \subset(0, \infty) \rightarrow \mathbb{R}$ be a function such that $f \in L[a, b]$, where $a, b \in I$ with $a<b$. If $f$ is a harmonically convex function on $[a, b]$, then the following inequalities for fractional integrals holds:

$$
f\left(\frac{2 a b}{a+b}\right) \leq \frac{\Gamma(\alpha+1)}{2}\left(\frac{a b}{b-a}\right)^{\alpha}\left[J_{1 / a-}^{\alpha}(f \circ h)(1 / b)+J_{1 / b+}^{\alpha}(f \circ h)(1 / a)\right] \leq \frac{f(a)+f(b)}{2}
$$

with $\alpha>0$ and $h(x)=1 / x$.

In [13] Latif et. al. gave the following definition.

Definition 3. A function $g:[a, b] \subseteq \mathbb{R} \backslash\{0\} \rightarrow \mathbb{R}$ is said to be harmonically symmetric with respect to $2 a b / a+b$ if

$$
g(x)=g\left(\frac{1}{\frac{1}{a}+\frac{1}{b}-\frac{1}{x}}\right)
$$

holds for all $x \in[a, b]$.

In [2] Chan and Wu presented Hermite-Hadamard-Fejér inequality for harmonically convex functions as follows.

Theorem 4. Let $f: I \subseteq \mathbb{R} \backslash\{0\} \rightarrow \mathbb{R}$ be a harmonically convex function and $a, b \in I$ with $a<b$. If $f \in L[a, b]$ and $g:[a, b] \subseteq \mathbb{R} \backslash\{0\} \rightarrow \mathbb{R}$ is nonnegative, integrable and harmonically symmetric with respect to $\frac{2 a b}{a+b}$, then

$$
\begin{aligned}
f\left(\frac{2 a b}{a+b}\right) \int_{a}^{b} \frac{g(x)}{x^{2}} d x & \leq \int_{a}^{b} \frac{f(x) g(x)}{x^{2}} d x \\
& \leq \frac{f(a)+f(b)}{2} \int_{a}^{b} \frac{g(x)}{x^{2}} d x
\end{aligned}
$$

In this paper, we firstly presented Hermite-Hadamard-Fejér inequality for harmonically convex function in fractional integral forms which is the weighted generalization of Hermite-Hadamard inequality for harmonically convex functions (5). Secondly, we obtained some new inequalities connected with the right-hand side of Hermite-Hadamard-Fejér type integral inequality for harmonically convex function in fractional integrals.

\section{Main results}

Throughout this section, let $\|g\|_{\infty}=\sup _{t \in[a, b]}|g(t)|$, for the continuous function $g:[a, b] \rightarrow \mathbb{R}$.

Lemma 2. If $g:[a, b] \subseteq \mathbb{R} \backslash\{0\} \rightarrow \mathbb{R}$ is integrable and harmonically symmetric with respect to $2 a b / a+b$ with $a<b$, then

$$
J_{1 / b+}^{\alpha}(g \circ h)(1 / a)=J_{1 / a-}^{\alpha}(g \circ h)(1 / b)=\frac{1}{2}\left[J_{1 / b+}^{\alpha}(g \circ h)(1 / a)+J_{1 / a-}^{\alpha}(g \circ h)(1 / b)\right]
$$

with $\alpha>0$ and $h(x)=1 / x, x \in\left[\frac{1}{b}, \frac{1}{a}\right]$.

Proof. Since $g$ is harmonically symmetric with respect to $2 a b / a+b$, from Definition 3 we have $g\left(\frac{1}{x}\right)=g\left(\frac{1}{\frac{1}{a}+\frac{1}{b}-x}\right)$, for all $x \in\left[\frac{1}{b}, \frac{1}{a}\right]$. Setting $t=\frac{1}{a}+\frac{1}{b}-x$ and $d t=-d x$ gives

$$
\begin{aligned}
J_{1 / b+}^{\alpha}(g \circ h)(1 / a) & =\frac{1}{\Gamma(\alpha)} \int_{\frac{1}{b}}^{\frac{1}{a}}\left(\frac{1}{a}-t\right)^{\alpha-1} g\left(\frac{1}{t}\right) d t=\frac{1}{\Gamma(\alpha)} \int_{\frac{1}{b}}^{\frac{1}{a}}\left(x-\frac{1}{b}\right)^{\alpha-1} g\left(\frac{1}{\frac{1}{a}+\frac{1}{b}-x}\right) d x \\
& =\frac{1}{\Gamma(\alpha)} \int_{\frac{1}{b}}^{\frac{1}{a}}\left(x-\frac{1}{b}\right)^{\alpha-1} g\left(\frac{1}{x}\right) d x=J_{1 / a-}^{\alpha}(g \circ h)(1 / b) .
\end{aligned}
$$

This completes the proof. 
Theorem 5. Let $f:[a, b] \rightarrow \mathbb{R}$ be a harmonically convex function with $a<b$ and $f \in L[a, b]$. If $g:[a, b] \rightarrow \mathbb{R}$ is nonnegative, integrable and harmonically symmetric with respect to $2 a b / a+b$, then the following inequalities for fractional integrals holds:

$$
f\left(\frac{2 a b}{a+b}\right)\left[\begin{array}{c}
J_{1 / b+}^{\alpha}(g \circ h)(1 / a) \\
+J_{1 / a-}^{\alpha}(g \circ h)(1 / b)
\end{array}\right] \leq\left[\begin{array}{c}
J_{1 / b+}^{\alpha}(f g \circ h)(1 / a) \\
+J_{1 / a-}^{\alpha}(f g \circ h)(1 / b)
\end{array}\right] \leq \frac{f(a)+f(b)}{2}\left[\begin{array}{c}
J_{1 / b+}^{\alpha}(g \circ h)(1 / a) \\
+J_{1 / a-}^{\alpha}(g \circ h)(1 / b)
\end{array}\right]
$$

with $\alpha>0$ and $h(x)=1 / x, x \in\left[\frac{1}{b}, \frac{1}{a}\right]$.

Proof. Since $f$ is a harmonically convex function on $[a, b]$, we write

$$
f\left(\frac{2 a b}{a+b}\right)=f\left(\frac{2\left(\frac{a b}{t a+(1-t) b}\right)\left(\frac{a b}{t b+(1-t) a}\right)}{\left(\frac{a b}{t a+(1-t) b}\right)+\left(\frac{a b}{t b+(1-t) a}\right)}\right) \leq \frac{f\left(\frac{a b}{t a+(1-t) b}\right)+f\left(\frac{a b}{t b+(1-t) a}\right)}{2} .
$$

for all $t \in[0,1]$. Multiplying both sides of (8) by $2 t^{\alpha-1} g\left(\frac{a b}{t b+(1-t) a}\right)$ then integrating the resulting inequality with respect to $t$ over $[0,1]$, we have

$$
\begin{aligned}
& 2 f\left(\frac{2 a b}{a+b}\right) \int_{0}^{1} t^{\alpha-1} g\left(\frac{a b}{t b+(1-t) a}\right) d t \\
& \leq \int_{0}^{1} t^{\alpha-1}\left[f\left(\frac{a b}{t a+(1-t) b}\right)+f\left(\frac{a b}{t b+(1-t) a}\right)\right] g\left(\frac{a b}{t b+(1-t) a}\right) d t \\
& =\int_{0}^{1} t^{\alpha-1} f\left(\frac{a b}{t a+(1-t) b}\right) g\left(\frac{a b}{t b+(1-t) a}\right) d t+\int_{0}^{1} t^{\alpha-1} f\left(\frac{a b}{t b+(1-t) a}\right) g\left(\frac{a b}{t b+(1-t) a}\right) d t
\end{aligned}
$$

Since $g$ is harmonically symmetric with respect to $2 a b / a+b$, from Definition 3, we have $g\left(\frac{1}{x}\right)=g\left(\frac{1}{\left(\frac{1}{a}\right)+\left(\frac{1}{b}\right)-x}\right)$, for all $x \in\left[\frac{1}{b}, \frac{1}{a}\right]$. Setting $x=\frac{t b+(1-t) a}{a b}$, and $d x=\left(\frac{b-a}{a b}\right) d t$ gives

$$
\begin{aligned}
& 2\left(\frac{a b}{b-a}\right)^{\alpha} f\left(\frac{2 a b}{a+b}\right) \int_{\frac{1}{b}}^{\frac{1}{a}}\left(x-\frac{1}{b}\right)^{\alpha-1} g\left(\frac{1}{x}\right) d x \\
& \leq\left(\frac{a b}{b-a}\right)^{\alpha}\left\{\int_{\frac{1}{b}}^{\frac{1}{a}}\left(x-\frac{1}{b}\right)^{\alpha-1} f\left(\frac{1}{\frac{1}{a}+\frac{1}{b}-x}\right) g\left(\frac{1}{x}\right) d x+\int_{\frac{1}{b}}^{\frac{1}{a}}\left(x-\frac{1}{b}\right)^{\alpha-1} f\left(\frac{1}{x}\right) g\left(\frac{1}{x}\right) d x\right\} \\
& =\left(\frac{a b}{b-a}\right)^{\alpha}\left\{\int_{\frac{1}{b}}^{\frac{1}{a}}\left(\frac{1}{a}-x\right)^{\alpha-1} f\left(\frac{1}{x}\right) g\left(\frac{1}{\frac{1}{a}+\frac{1}{b}-x}\right) d x+\int_{\frac{1}{b}}^{\frac{1}{a}}\left(x-\frac{1}{b}\right)^{\alpha-1} f\left(\frac{1}{x}\right) g\left(\frac{1}{x}\right) d x\right\} \\
& =\left(\frac{a b}{b-a}\right)^{\alpha}\left\{\int_{\frac{1}{b}}^{\frac{1}{a}}\left(\frac{1}{a}-x\right)^{\alpha-1} f\left(\frac{1}{x}\right) g\left(\frac{1}{x}\right) d x+\int_{\frac{1}{b}}^{\frac{1}{a}}\left(x-\frac{1}{b}\right)^{\alpha-1} f\left(\frac{1}{x}\right) g\left(\frac{1}{x}\right) d x\right\} .
\end{aligned}
$$

Using Lemma 2, we have

$$
\left(\frac{a b}{b-a}\right)^{\alpha} \Gamma(\alpha) f\left(\frac{2 a b}{a+b}\right)\left[\begin{array}{c}
J_{1 / b+}^{\alpha}(g \circ h)(1 / a) \\
+J_{1 / a-}^{\alpha}(g \circ h)(1 / b)
\end{array}\right] \leq\left(\frac{a b}{b-a}\right)^{\alpha} \Gamma(\alpha)\left[\begin{array}{c}
J_{1 / b+}^{\alpha}(f g \circ h)(1 / a) \\
+J_{1 / a-}^{\alpha}(f g \circ h)(1 / b)
\end{array}\right] .
$$

This inequality gives the left hand side of (7).

On the other hand, since $f$ is a harmonically convex function, then, for all $t \in[0,1]$, we have

$$
f\left(\frac{a b}{t a+(1-t) b}\right)+f\left(\frac{a b}{t b+(1-t) a}\right) \leq f(a)+f(b)
$$


Then multiplying both sides of (9) by $t^{\alpha-1} g\left(\frac{a b}{t b+(1-t) a}\right)$ and integrating the resulting inequality with respect to $t$ over $[0,1]$, we have

$$
\begin{aligned}
& \int_{0}^{1} t^{\alpha-1} f\left(\frac{a b}{t a+(1-t) b}\right) g\left(\frac{a b}{t b+(1-t) a}\right) d t+\int_{0}^{1} t^{\alpha-1} f\left(\frac{a b}{t b+(1-t) a}\right) g\left(\frac{a b}{t b+(1-t) a}\right) d t \\
& \leq[f(a)+f(b)] \int_{0}^{1} t^{\alpha-1} g\left(\frac{a b}{t b+(1-t) a}\right) d t .
\end{aligned}
$$

It means that

$$
\left(\frac{a b}{b-a}\right)^{\alpha} \Gamma(\alpha)\left[\begin{array}{c}
J_{1 / b+}^{\alpha}(f g \circ h)(1 / a) \\
+J_{1 / a-}^{\alpha}(f g \circ h)(1 / b)
\end{array}\right] \leq\left(\frac{a b}{b-a}\right)^{\alpha} \Gamma(\alpha)\left(\frac{f(a)+f(b)}{2}\right)\left[\begin{array}{c}
J_{1 / b+}^{\alpha}(g \circ h)(1 / a) \\
+J_{1 / a-}^{\alpha}(g \circ h)(1 / b)
\end{array}\right] .
$$

This inequality gives the right hand side of (7). The proof is completed.

Remark. In Theorem 5, one can see the following.

(i) If one takes $\alpha=1$, then inequality (7) becomes inequality (6) of Theorem 4.

(ii) If one takes $g(x)=1$, then inequality (7) becomes inequality (5) of Theorem 3 .

(iii) If one takes $\alpha=1$ and $g(x)=1$, then inequality (7) becomes inequality (4) of Theorem 2 .

Lemma 3. Let $f: I \subset(0, \infty) \rightarrow \mathbb{R}$ be a differentiable function on $I^{\circ}$ such that $f^{\prime} \in L[a, b]$, where $a, b \in I$ and $a<b$. If $g:[a, b] \rightarrow \mathbb{R}$ is integrable and harmonically symmetric with respect to $2 a b / a+b$, then the following equality for fractional integrals holds

$$
\begin{aligned}
& \frac{f(a)+f(b)}{2}\left[J_{1 / b+}^{\alpha}(g \circ h)(1 / a)+J_{1 / a-}^{\alpha}(g \circ h)(1 / b)\right]-\left[J_{1 / b+}^{\alpha}(f g \circ h)(1 / a)+J_{1 / a-}^{\alpha}(f g \circ h)(1 / b)\right] \\
& =\frac{1}{\Gamma(\alpha)} \int_{\frac{1}{b}}^{\frac{1}{a}}\left[\int_{\frac{1}{b}}^{t}\left(\frac{1}{a}-s\right)^{\alpha-1}(g \circ h)(s) d s-\int_{t}^{\frac{1}{a}}\left(s-\frac{1}{b}\right)^{\alpha-1}(g \circ h)(s) d s\right](f \circ h)^{\prime}(t) d t
\end{aligned}
$$

with $\alpha>0$ and $h(x)=1 / x, x \in\left[\frac{1}{b}, \frac{1}{a}\right]$.

Proof. It suffices to note that

$$
\begin{aligned}
I & =\int_{\frac{1}{b}}^{\frac{1}{a}}\left[\begin{array}{c}
\int_{\frac{1}{b}}^{t}\left(\frac{1}{a}-s\right)^{\alpha-1}(g \circ h)(s) d s \\
-\int_{t}^{\frac{1}{a}}\left(s-\frac{1}{b}\right)^{\alpha-1}(g \circ h)(s) d s
\end{array}\right](f \circ h)^{\prime}(t) d t \\
& =\int_{\frac{1}{b}}^{\frac{1}{a}}\left[\int_{\frac{1}{b}}^{t}\left(\frac{1}{a}-s\right)^{\alpha-1}(g \circ h)(s) d s\right](f \circ h)^{\prime}(t) d t-\int_{\frac{1}{b}}^{\frac{1}{a}}\left[\int_{t}^{\frac{1}{a}}\left(s-\frac{1}{b}\right)^{\alpha-1}(g \circ h)(s) d s\right](f \circ h)^{\prime}(t) d t \\
& =I_{1}-I_{2} .
\end{aligned}
$$

By integration by parts and using Lemma 2, we have

$$
\begin{aligned}
I_{1} & =\left.\left(\int_{\frac{1}{b}}^{t}\left(\frac{1}{a}-s\right)^{\alpha-1}(g \circ h)(s) d s\right)(f \circ h)(t)\right|_{\frac{1}{b}} ^{\frac{1}{a}}-\int_{\frac{1}{b}}^{\frac{1}{a}}\left(\frac{1}{a}-t\right)^{\alpha-1}(g \circ h)(t)(f \circ h)(t) d t \\
& =\left(\int_{\frac{1}{b}}^{\frac{1}{a}}\left(\frac{1}{a}-s\right)^{\alpha-1}(g \circ h)(s) d s\right) f(a)-\int_{\frac{1}{b}}^{\frac{1}{a}}\left(\frac{1}{a}-t\right)^{\alpha-1}(g \circ h)(t)(f \circ h)(t) d t \\
& =\Gamma(\alpha)\left[f(a) J_{1 / b+}^{\alpha}(g \circ h)(1 / a)-J_{1 / b+}^{\alpha}(f g \circ h)(1 / a)\right] \\
& =\Gamma(\alpha)\left[\frac{f(a)}{2}\left[J_{1 / b+}^{\alpha}(g \circ h)(1 / a)+J_{1 / a-}^{\alpha}(g \circ h)(1 / b)\right]-J_{1 / b+}^{\alpha}(f g \circ h)(1 / a)\right] .
\end{aligned}
$$


Similarly we have

$$
\begin{aligned}
I_{2} & =\left.\left(\int_{t}^{\frac{1}{a}}\left(s-\frac{1}{b}\right)^{\alpha-1}(g \circ h)(s) d s\right)(f \circ h)(t)\right|_{\frac{1}{b}} ^{\frac{1}{a}}-\int_{\frac{1}{b}}^{\frac{1}{a}}\left(t-\frac{1}{b}\right)^{\alpha-1}(g \circ h)(t)(f \circ h)(t) d t \\
& =-\left(\int_{\frac{1}{b}}^{\frac{1}{a}}\left(s-\frac{1}{b}\right)^{\alpha-1}(g \circ h)(s) d s\right) f(b)+\int_{\frac{1}{b}}^{\frac{1}{a}}\left(t-\frac{1}{b}\right)^{\alpha-1}(g \circ h)(t)(f \circ h)(t) d t \\
& =\Gamma(\alpha)\left[-f(b) J_{1 / a-}^{\alpha}(g \circ h)(1 / b)+J_{1 / a-}^{\alpha}(f g \circ h)(1 / b)\right] \\
& =\Gamma(\alpha)\left[-\frac{f(b)}{2}\left[J_{1 / b+}^{\alpha}(g \circ h)(1 / a)+J_{1 / a-}^{\alpha}(g \circ h)(1 / b)\right]+J_{1 / a-}^{\alpha}(f g \circ h)(1 / b)\right] .
\end{aligned}
$$

A combination of (11), (12) and (13) gives

$$
I=I_{1}-I_{2}=\Gamma(\alpha)\left\{\left(\frac{f(a)+f(b)}{2}\right)\left[\begin{array}{c}
J_{1 / b+}^{\alpha}(g \circ h)(1 / a) \\
+J_{1 / a-}^{\alpha}(g \circ h)(1 / b)
\end{array}\right]-\left[\begin{array}{c}
J_{1 / b+}^{\alpha}(f g \circ h)(1 / a) \\
+J_{1 / a-}^{\alpha}(f g \circ h)(1 / b)
\end{array}\right]\right\} .
$$

Multiplying both sides of (14) by $(\Gamma(\alpha))^{-1}$ we have (10). This completes the proof.

Remark. In Lemma 3, if one takes $g(x)=1$, then equality (10) becomes equality in [10, Lemma 3].

Theorem 6. Let $f: I \subset(0, \infty) \rightarrow \mathbb{R}$ be a differentiable function on $I^{\circ}$ such that $f^{\prime} \in L[a, b]$, where $a, b \in I$ and $a<b$. If $\left|f^{\prime}\right|$ is harmonically convex on $[a, b], g:[a, b] \rightarrow \mathbb{R}$ is continuous and harmonically symmetric with respect to $2 a b / a+b$, then the following inequality for fractional integrals holds.

$$
\begin{aligned}
& \left|\frac{f(a)+f(b)}{2}\left[J_{1 / b+}^{\alpha}(g \circ h)(1 / a)+J_{1 / a-}^{\alpha}(g \circ h)(1 / b)\right]-\left[J_{1 / b+}^{\alpha}(f g \circ h)(1 / a)+J_{1 / a-}^{\alpha}(f g \circ h)(1 / b)\right]\right| \\
& \leq \frac{\|g\|_{\infty} a b(b-a)}{\Gamma(\alpha+1)}\left(\frac{b-a}{a b}\right)^{\alpha}\left[C_{1}(\alpha)\left|f^{\prime}(a)\right|+C_{2}(\alpha)\left|f^{\prime}(b)\right|\right]
\end{aligned}
$$

where

$$
\begin{gathered}
C_{1}(\alpha)=\left[\begin{array}{c}
\frac{b^{-2}}{\alpha+2}{ }_{2} F_{1}\left(2,1 ; \alpha+3 ; 1-\frac{a}{b}\right) \\
-\frac{b^{-2}}{(\alpha+1)(\alpha+2)}{ }_{2} F_{1}\left(2, \alpha+1 ; \alpha+3 ; 1-\frac{a}{b}\right) \\
+\frac{2(a+b)^{-2}}{(\alpha+1)(\alpha+2)}{ }_{2} F_{1}\left(2, \alpha+1 ; \alpha+3 ; \frac{b-a}{b+a}\right)
\end{array}\right], \\
C_{2}(\alpha)=\left[\begin{array}{c}
\frac{b^{-2}}{(\alpha+1)(\alpha+2)}{ }_{2} F_{1}\left(2,2 ; \alpha+3 ; 1-\frac{a}{b}\right) \\
-\frac{b^{-2}}{\alpha+2}{ }_{2} F_{1}\left(2, \alpha+2 ; \alpha+3 ; 1-\frac{a}{b}\right) \\
+\left(\frac{a+b}{2}\right)^{-2} \frac{1}{\alpha+1}{ }_{2} F_{1}\left(2, \alpha+1 ; \alpha+2 ; \frac{b-a}{b+a}\right) \\
-\frac{2(a+b)^{-2}}{(\alpha+1)(\alpha+2)}{ }_{2} F_{1}\left(2, \alpha+1 ; \alpha+3 ; \frac{b-a}{b+a}\right)
\end{array}\right]
\end{gathered}
$$

with $0<\alpha \leq 1$ and $h(x)=1 / x, x \in\left[\frac{1}{b}, \frac{1}{a}\right]$.

Proof. From Lemma 3 we have

$$
\begin{aligned}
& \left|\frac{f(a)+f(b)}{2}\left[J_{1 / b+}^{\alpha}(g \circ h)(1 / a)+J_{1 / a-}^{\alpha}(g \circ h)(1 / b)\right]-\left[J_{1 / b+}^{\alpha}(f g \circ h)(1 / a)+J_{1 / a-}^{\alpha}(f g \circ h)(1 / b)\right]\right| \\
& \leq \frac{1}{\Gamma(\alpha)} \int_{\frac{1}{b}}^{\frac{1}{a}}\left|\int_{\frac{1}{b}}^{t}\left(\frac{1}{a}-s\right)^{\alpha-1}(g \circ h)(s) d s-\int_{t}^{\frac{1}{a}}\left(s-\frac{1}{b}\right)^{\alpha-1}(g \circ h)(s) d s\right|\left|(f \circ h)^{\prime}(t)\right| d t .
\end{aligned}
$$


Since $g$ is harmonically symmetric with respect to $2 a b / a+b$, using Definition 3 we have $g\left(\frac{1}{x}\right)=g\left(\frac{1}{\left(\frac{1}{a}\right)+\left(\frac{1}{b}\right)-x}\right)$, for all $x \in\left[\frac{1}{b}, \frac{1}{a}\right]$.

$$
\begin{aligned}
& \left|\int_{\frac{1}{b}}^{t}\left(\frac{1}{a}-s\right)^{\alpha-1}(g \circ h)(s) d s-\int_{t}^{\frac{1}{a}}\left(s-\frac{1}{b}\right)^{\alpha-1}(g \circ h)(s) d s\right| \\
& =\left|\int_{\frac{1}{a}+\frac{1}{b}-t}^{\frac{1}{a}}\left(s-\frac{1}{b}\right)^{\alpha-1}(g \circ h)(s) d s+\int_{\frac{1}{a}}^{t}\left(s-\frac{1}{b}\right)^{\alpha-1}(g \circ h)(s) d s\right| \\
& =\left|\int_{\frac{1}{a}+\frac{1}{b}-t}^{t}\left(s-\frac{1}{b}\right)^{\alpha-1}(g \circ h)(s) d s\right| \\
& \leq\left\{\begin{array}{l}
\int_{t}^{\frac{1}{a}+\frac{1}{b}-t}\left|\left(s-\frac{1}{b}\right)^{\alpha-1}(g \circ h)(s)\right| d s, t \in\left[\frac{1}{b}, \frac{a+b}{2 a b}\right] \\
\int_{\frac{1}{a}+\frac{1}{b}-t}^{t}\left|\left(s-\frac{1}{b}\right)^{\alpha-1}(g \circ h)(s)\right| d s, t \in\left[\frac{a+b}{2 a b}, \frac{1}{a}\right]
\end{array}\right.
\end{aligned}
$$

If we use (17) in (16), we have

$$
\begin{aligned}
& \mid \frac{f(a)+f(b)}{2}\left[J_{1 / b+}^{\alpha}(g \circ h)(1 / a)+J_{1 / a-}^{\alpha}(g \circ h)(1 / b)\right] \\
& -\left[J_{1 / b+}^{\alpha}(f g \circ h)(1 / a)+J_{1 / a-}^{\alpha}(f g \circ h)(1 / b)\right] \mid \\
& \leq \frac{1}{\Gamma(\alpha)}\left[\int_{\frac{1}{b}}^{\frac{a+b}{2 a b}}\left(\int_{t}^{\frac{1}{a}+\frac{1}{b}-t}\left|\left(s-\frac{1}{b}\right)^{\alpha-1}(g \circ h)(s)\right| d s\right)\left|(f \circ h)^{\prime}(t)\right| d t\right] \\
& \left.+\int_{\frac{a+b}{2 a b}}^{\frac{1}{a}}\left(\int_{\frac{1}{a}+\frac{1}{b}-t}^{t}\left|\left(s-\frac{1}{b}\right)^{\alpha-1}(g \circ h)(s)\right| d s\right)\left|(f \circ h)^{\prime}(t)\right| d t\right] \\
& \leq \frac{\|g\|_{\infty}}{\Gamma(\alpha)}\left[\int_{\frac{1}{b}}^{\frac{a+b}{2 a b}}\left(\int_{t}^{\frac{1}{a}+\frac{1}{b}-t}\left(s-\frac{1}{b}\right)^{\alpha-1} d s\right) \frac{1}{t^{2}}\left|f^{\prime}\left(\frac{1}{t}\right)\right| d t\right. \\
& \left.+\int_{\frac{a+b}{2 a b}}^{\frac{1}{a}}\left(\int_{\frac{1}{a}+\frac{1}{b}-t}^{t}\left(s-\frac{1}{b}\right)^{\alpha-1} d s\right) \frac{1}{t^{2}}\left|f^{\prime}\left(\frac{1}{t}\right)\right| d t\right] .
\end{aligned}
$$

Setting $t=\frac{u b+(1-u) a}{a b}$, and $d t=\left(\frac{b-a}{a b}\right) d u$ gives

$$
\begin{aligned}
& \mid \frac{f(a)+f(b)}{2}\left[J_{1 / b+}^{\alpha}(g \circ h)(1 / a)+J_{1 / a-}^{\alpha}(g \circ h)(1 / b)\right] \\
& -\left[J_{1 / b+}^{\alpha}(f g \circ h)(1 / a)+J_{1 / a-}^{\alpha}(f g \circ h)(1 / b)\right] \mid \\
& \leq \frac{\|g\|_{\infty} a b(b-a)}{\Gamma(\alpha+1)}\left(\frac{b-a}{a b}\right)^{\alpha}\left[\begin{array}{c}
\int_{0}^{\frac{1}{2}} \frac{(1-u)^{\alpha}-u^{\alpha}}{(u b+(1-u) a)^{2}}\left|f^{\prime}\left(\frac{a b}{u b+(1-u) a}\right)\right| d u \\
+\int_{\frac{1}{2}}^{1} \frac{u^{\alpha}-(1-u)^{\alpha}}{(u b+(1-u) a)^{2}}\left|f^{\prime}\left(\frac{a b}{u b+(1-u) a}\right)\right| d u
\end{array}\right] .
\end{aligned}
$$

Since $\left|f^{\prime}\right|$ is harmonically convex on $[a, b]$, we have

$$
\left|f^{\prime}\left(\frac{a b}{u b+(1-u) a}\right)\right| \leq u\left|f^{\prime}(a)\right|+(1-u)\left|f^{\prime}(b)\right| .
$$


If we use (19) in (18), we have

$$
\begin{aligned}
& \left|\frac{f(a)+f(b)}{2}\left[J_{1 / b+}^{\alpha}(g \circ h)(1 / a)+J_{1 / a-}^{\alpha}(g \circ h)(1 / b)\right]-\left[J_{1 / b+}^{\alpha}(f g \circ h)(1 / a)+J_{1 / a-}^{\alpha}(f g \circ h)(1 / b)\right]\right| \\
& \leq \frac{\|g\|_{\infty} a b(b-a)}{\Gamma(\alpha+1)}\left(\frac{b-a}{a b}\right)^{\alpha}\left[\begin{array}{l}
{\left[\int_{0}^{\frac{1}{2}} \frac{(1-u)^{\alpha}-u^{\alpha}}{(u b+(1-u) a)^{2}} u d u+\int_{\frac{1}{2}}^{1} \frac{u^{\alpha}-(1-u)^{\alpha}}{(u b+(1-u) a)^{2}} u d u\right]\left|f^{\prime}(a)\right|} \\
\left.+\left[\int_{0}^{\frac{1}{2}} \frac{(1-u)^{\alpha}-u^{\alpha}}{(u b+(1-u) a)^{2}}(1-u) d u+\int_{\frac{1}{2}}^{1} \frac{u^{\alpha}-(1-u)^{\alpha}}{(u b+(1-u) a)^{2}}(1-u) d u\right]\left|f^{\prime}(b)\right|\right]
\end{array}\right.
\end{aligned}
$$

Using Lemma 1, we have

$$
\begin{aligned}
& \int_{0}^{\frac{1}{2}} \frac{(1-u)^{\alpha}-u^{\alpha}}{(u b+(1-u) a)^{2}} u d u+\int_{\frac{1}{2}}^{1} \frac{u^{\alpha}-(1-u)^{\alpha}}{(u b+(1-u) a)^{2}} u d u \\
& =\int_{0}^{1} \frac{u^{\alpha}-(1-u)^{\alpha}}{(u b+(1-u) a)^{2}} u d u+2 \int_{0}^{\frac{1}{2}} \frac{(1-u)^{\alpha}-u^{\alpha}}{(u b+(1-u) a)^{2}} u d u \\
& =\int_{0}^{1} \frac{u^{\alpha+1}}{(u b+(1-u) a)^{2}} d u-\int_{0}^{1} \frac{u(1-u)^{\alpha}}{(u b+(1-u) a)^{2}} d u+2 \int_{0}^{\frac{1}{2}} \frac{(1-u)^{\alpha}-u^{\alpha}}{(u b+(1-u) a)^{2}} u d u \\
& \leq \int_{0}^{1} \frac{u^{\alpha+1}}{(u b+(1-u) a)^{2}} d u-\int_{0}^{1} \frac{u(1-u)^{\alpha}}{(u b+(1-u) a)^{2}} d u+2 \int_{0}^{\frac{1}{2}} \frac{(1-2 u)^{\alpha}}{(u b+(1-u) a)^{2}} u d u
\end{aligned}
$$

and

$$
\begin{aligned}
& \int_{0}^{\frac{1}{2}} \frac{(1-u)^{\alpha}-u^{\alpha}}{(u b+(1-u) a)^{2}}(1-u) d u+\int_{\frac{1}{2}}^{1} \frac{u^{\alpha}-(1-u)^{\alpha}}{(u b+(1-u) a)^{2}}(1-u) d u \\
& =\int_{0}^{1} \frac{u^{\alpha}-(1-u)^{\alpha}}{(u b+(1-u) a)^{2}}(1-u) d u+2 \int_{0}^{\frac{1}{2}} \frac{(1-u)^{\alpha}-u^{\alpha}}{(u b+(1-u) a)^{2}}(1-u) d u \\
& \leq \int_{0}^{1} \frac{u(1-u)^{\alpha}}{(u a+(1-u) b)^{2}} d u-\int_{0}^{1} \frac{u^{\alpha+1}}{(u a+(1-u) b)^{2}} d u \\
& +\int_{0}^{1} \frac{(1-u)^{\alpha}}{\left(\frac{u}{2} b+\left(1-\frac{u}{2}\right) a\right)^{2}} d u-\frac{1}{2} \int_{0}^{1} \frac{u(1-u)^{\alpha}}{\left(\frac{u}{2} b+\left(1-\frac{u}{2}\right) a\right)^{2}} d u
\end{aligned}
$$

Calculating following integrals, we have

$$
\begin{aligned}
& \int_{0}^{1} \frac{(1-u)^{\alpha+1}}{(u a+(1-u) b)^{2}} d u-\int_{0}^{1} \frac{(1-u) u^{\alpha}}{(u a+(1-u) b)^{2}} d u+\frac{1}{2} \int_{0}^{1} \frac{u(1-u)^{\alpha}}{\left(\frac{u}{2} b+\left(1-\frac{u}{2}\right) a\right)^{2}} d u \\
& =\int_{0}^{1}(1-u)^{\alpha+1} b^{-2}\left(1-u\left(1-\frac{a}{b}\right)\right)^{-2} d u-\int_{0}^{1}(1-u) u^{\alpha} b^{-2}\left(1-u\left(1-\frac{a}{b}\right)\right)^{-2} d u \\
& +\frac{1}{2} \int_{0}^{1}(1-v) v^{\alpha}\left(\frac{a+b}{2}\right)^{-2}\left(1-v\left(\frac{b-a}{b+a}\right)\right)^{-2} d v=\left[\begin{array}{c}
\frac{b^{-2}}{\alpha+2}{ }_{2} F_{1}\left(2,1 ; \alpha+3 ; 1-\frac{a}{b}\right) \\
-\frac{b^{-2}}{(\alpha+1)(\alpha+2)}{ }_{2} F_{1}\left(2, \alpha+1 ; \alpha+3 ; 1-\frac{a}{b}\right) \\
+\frac{2(a+b)^{-2}}{(\alpha+1)(\alpha+2)}{ }_{2} F_{1}\left(2, \alpha+1 ; \alpha+3 ; \frac{b-a}{b+a}\right)
\end{array}\right]=C_{1}(\alpha)
\end{aligned}
$$


and

$$
\begin{aligned}
& \int_{0}^{1} \frac{u(1-u)^{\alpha}}{(u a+(1-u) b)^{2}} d u-\int_{0}^{1} \frac{u^{\alpha+1}}{(u a+(1-u) b)^{2}} d u+\int_{0}^{1} \frac{(1-u)^{\alpha}}{\left(\frac{u}{2} b+\left(1-\frac{u}{2}\right) a\right)^{2}} d u-\frac{1}{2} \int_{0}^{1} \frac{u(1-u)^{\alpha}}{\left(\frac{u}{2} b+\left(1-\frac{u}{2}\right) a\right)^{2}} d u \\
& =\int_{0}^{1} \frac{u(1-u)^{\alpha}}{(u a+(1-u) b)^{2}} d u-\int_{0}^{1} \frac{u^{\alpha+1}}{(u a+(1-u) b)^{2}} d u+\left(\frac{a+b}{2}\right)^{-2} \int_{0}^{1} v^{\alpha}\left(1-v\left(\frac{b-a}{b+a}\right)\right)^{-2} d v \\
& -\frac{1}{2}\left(\frac{a+b}{2}\right)^{-2} \int_{0}^{1}(1-v) v^{\alpha}\left(1-v\left(\frac{b-a}{b+a}\right)\right)^{-2} d v=\left[\begin{array}{c}
\frac{b^{-2}}{(\alpha+1)(\alpha+2)}{ }_{2} F_{1}\left(2,2 ; \alpha+3 ; 1-\frac{a}{b}\right) \\
-\frac{b^{-2}}{\alpha+2}{ }_{2} F_{1}\left(2, \alpha+2 ; \alpha+3 ; 1-\frac{a}{b}\right) \\
+\left(\frac{a+b}{2}\right)^{-2} \frac{1}{\alpha+1}{ }_{2} F_{1}\left(2, \alpha+1 ; \alpha+2 ; \frac{b-a}{b+a}\right) \\
-\frac{2(a+b)^{-2}}{(\alpha+1)(\alpha+2)}{ }_{2} F_{1}\left(2, \alpha+1 ; \alpha+3 ; \frac{b-a}{b+a}\right)
\end{array}\right]=C_{2}(\alpha) \text {. }
\end{aligned}
$$

If we use (21), (22), (23) and (24) in (20), we have (15). This completes the proof.

Corollary 1. In Theorem 6, one has the following.

(1) If one takes $\alpha=1$, one has the following Hermite-Hadamard-Fejér inequality for harmonically convex functions which is related the right-hand side of (6):

$$
\left|\frac{f(a)+f(b)}{2} \int_{a}^{b} \frac{g(x)}{x^{2}} d x-\int_{a}^{b} \frac{f(x) g(x)}{x^{2}} d x\right| \leq \frac{\|g\|_{\infty}(b-a)^{2}}{2}\left[C_{1}(1)\left|f^{\prime}(a)\right|+C_{2}(1)\left|f^{\prime}(b)\right|\right],
$$

(2) If one takes $g(x)=1$, one has the following Hermite-Hadamard type inequality for harmonically convex function in fractional integral forms which is related the right-hand side of (5):

$$
\left|\frac{f(a)+f(b)}{2}-\frac{\Gamma(\alpha+1)}{2}\left(\frac{a b}{b-a}\right)^{\alpha}\left\{\begin{array}{c}
J_{1 / a-}^{\alpha}(f \circ h)(1 / b) \\
+J_{1 / b+}^{\alpha}(f \circ h)(1 / a)
\end{array}\right\}\right| \leq \frac{a b(b-a)}{2}\left[C_{1}(\alpha)\left|f^{\prime}(a)\right|+C_{2}(\alpha)\left|f^{\prime}(b)\right|\right]
$$

(3) If one takes $\alpha=1$ and $g(x)=1$, one has the following Hermite-Hadamard type inequality for harmonically convex function which is related the right-hand side of (4):

$$
\left|\frac{f(a)+f(b)}{2}-\frac{a b}{b-a} \int_{a}^{b} \frac{f(x)}{x^{2}} d x\right| \leq \frac{a b(b-a)}{2}\left[C_{1}(1)\left|f^{\prime}(a)\right|+C_{2}(1)\left|f^{\prime}(b)\right|\right] .
$$

Theorem 7. Let $f: I \subset(0, \infty) \rightarrow \mathbb{R}$ be a differentiable function on $I^{\circ}$ such that $f^{\prime} \in L[a, b]$, where $a, b \in I$ and $a<b$. If $\left|f^{\prime}\right|^{q}, q \geq 1$, is harmonically convex on $[a, b], g:[a, b] \rightarrow \mathbb{R}$ is continuous and harmonically symmetric with respect to $2 a b / a+b$, then the following inequality for fractional integrals holds:

$$
\begin{aligned}
& \left|\frac{f(a)+f(b)}{2}\left[J_{1 / b+}^{\alpha}(g \circ h)(1 / a)+J_{1 / a-}^{\alpha}(g \circ h)(1 / b)\right]-\left[J_{1 / b+}^{\alpha}(f g \circ h)(1 / a)+J_{1 / a-}^{\alpha}(f g \circ h)(1 / b)\right]\right| \\
& \leq \frac{\|g\|_{\infty} a b(b-a)}{\Gamma(\alpha+1)}\left(\frac{b-a}{a b}\right)^{\alpha}\left[C_{3}^{1-\frac{1}{q}}(\alpha)\left[\left(\begin{array}{c}
C_{4}(\alpha)\left|f^{\prime}(a)\right|^{q} \\
+C_{5}(\alpha)\left|f^{\prime}(b)\right|^{q}
\end{array}\right)\right]^{\frac{1}{q}}+C_{6}^{1-\frac{1}{q}}(\alpha)\left[\left(\begin{array}{c}
C_{7}(\alpha)\left|f^{\prime}(a)\right|^{q} \\
+C_{8}(\alpha)\left|f^{\prime}(b)\right|^{q}
\end{array}\right)\right]^{\frac{1}{q}}\right]
\end{aligned}
$$


where

$$
\begin{aligned}
& C_{3}(\alpha)=\frac{2(a+b)^{-2}}{(\alpha+1)}{ }_{2} F_{1}\left(2, \alpha+1 ; \alpha+3 ; \frac{b-a}{b+a}\right), \\
& C_{4}(\alpha)=\frac{(a+b)^{-2}}{(\alpha+1)(\alpha+2)}{ }_{2} F_{1}\left(2, \alpha+1 ; \alpha+3 ; \frac{b-a}{b+a}\right), \\
& C_{5}(\alpha)=C_{3}(\alpha)-C_{4}(\alpha), \\
& C_{6}(\alpha)=\left[\begin{array}{c}
\frac{b^{-2}}{(\alpha+1)} 2{ }_{2} F_{1}\left(2,1 ; \alpha+2 ; 1-\frac{a}{b}\right) \\
-\frac{b^{-2}}{(\alpha+1)}{ }_{2} F_{1}\left(2, \alpha+1 ; \alpha+2 ; 1-\frac{a}{b}\right)+C_{3}(\alpha)
\end{array}\right], \\
& C_{7}(\alpha)=\left[\begin{array}{c}
\quad \frac{b^{-2}}{(\alpha+2)} 2{ }_{2} F_{1}\left(2,1 ; \alpha+3 ; 1-\frac{a}{b}\right) \\
-\frac{b^{-2}}{(\alpha+1)(\alpha+2)}{ }_{2} F_{1}\left(2, \alpha+1 ; \alpha+3 ; 1-\frac{a}{b}\right)+C_{4}(\alpha)
\end{array}\right], \\
& C_{8}(\alpha)=C_{6}(\alpha)-C_{7}(\alpha),
\end{aligned}
$$

with $0<\alpha \leq 1$ and $h(x)=1 / x, x \in\left[\frac{1}{b}, \frac{1}{a}\right]$.

Proof. By using power mean inequality and the harmonically convexity of $\left|f^{\prime}\right|^{q}$ in (18), we have

$$
\begin{aligned}
& \left|\frac{f(a)+f(b)}{2}\left[J_{1 / b+}^{\alpha}(g \circ h)(1 / a)+J_{1 / a-}^{\alpha}(g \circ h)(1 / b)\right]-\left[J_{1 / b+}^{\alpha}(f g \circ h)(1 / a)+J_{1 / a-}^{\alpha}(f g \circ h)(1 / b)\right]\right| \\
& \leq \frac{\|g\|_{\infty} a b(b-a)}{\Gamma(\alpha+1)}\left(\frac{b-a}{a b}\right)^{\alpha}\left[\begin{array}{c}
\int_{0}^{\frac{1}{2}} \frac{(1-u)^{\alpha}-u^{\alpha}}{(u b+(1-u) a)^{2}}\left|f^{\prime}\left(\frac{a b}{u b+(1-u) a}\right)\right| d u \\
+\int_{\frac{1}{2}}^{1} \frac{u^{\alpha}-(1-u)^{\alpha}}{(u b+(1-u) a)^{2}}\left|f^{\prime}\left(\frac{a b}{u b+(1-u) a}\right)\right| d u
\end{array}\right] \\
& \leq \frac{\|g\|_{\infty} a b(b-a)}{\Gamma(\alpha+1)}\left(\frac{b-a}{a b}\right)^{\alpha}\left[\left(\int_{0}^{\frac{1}{2}} \frac{(1-u)^{\alpha}-u^{\alpha}}{(u b+(1-u) a)^{2}} d u\right)^{1-\frac{1}{q}}\left(\int_{0}^{\frac{1}{2}} \frac{(1-u)^{\alpha}-u^{\alpha}}{(u b+(1-u) a)^{2}}\left|f^{\prime}\left(\frac{a b}{u b+(1-u) a}\right)\right|^{q} d u\right)^{\frac{1}{q}}\right. \\
& \left.+\left(\int_{\frac{1}{2}}^{1} \frac{u^{\alpha}-(1-u)^{\alpha}}{(u b+(1-u) a)^{2}} d u\right)^{1-\frac{1}{q}}\left(\int_{\frac{1}{2}}^{1} \frac{u^{\alpha}-(1-u)^{\alpha}}{(u b+(1-u) a)^{2}}\left|f^{\prime}\left(\frac{a b}{u b+(1-u) a}\right)\right|^{q} d u\right)^{\frac{1}{q}}\right] \\
& \leq \frac{\|g\|_{\infty} a b(b-a)}{\Gamma(\alpha+1)}\left(\frac{b-a}{a b}\right)^{\alpha}\left[\left(\int_{0}^{\frac{1}{2}} \frac{(1-u)^{\alpha}-u^{\alpha}}{(u b+(1-u) a)^{2}} d u\right)^{1-\frac{1}{q}}\left(\int_{0}^{\frac{1}{2}} \frac{(1-u)^{\alpha}-u^{\alpha}}{(u b+(1-u) a)^{2}}\left[u\left|f^{\prime}(a)\right|^{q}+(1-u)\left|f^{\prime}(b)\right|^{q}\right] d u\right)^{\frac{1}{q}}\right. \\
& \left.+\left(\int_{\frac{1}{2}}^{1} \frac{u^{\alpha}-(1-u)^{\alpha}}{(u b+(1-u) a)^{2}} d u\right)^{1-\frac{1}{q}}\left(\int_{\frac{1}{2}}^{1} \frac{u^{\alpha}-(1-u)^{\alpha}}{(u b+(1-u) a)^{2}}\left[u\left|f^{\prime}(a)\right|^{q}+(1-u)\left|f^{\prime}(b)\right|^{q}\right] d u\right)^{\frac{1}{q}}\right] \\
& \leq \frac{\|g\|_{\infty} a b(b-a)}{\Gamma(\alpha+1)}\left(\frac{b-a}{a b}\right)^{\alpha} \\
& \times\left[\begin{array}{l}
\left(\int_{0}^{\frac{1}{2}} \frac{(1-u)^{\alpha}-u^{\alpha}}{(u b+(1-u) a)^{2}} d u\right)^{1-\frac{1}{q}}\left(\int_{0}^{\frac{1}{2}} \frac{(1-u)^{\alpha}-u^{\alpha}}{(u b+(1-u) a)^{2}} u d u\left|f^{\prime}(a)\right|^{q} \int_{0}^{\frac{1}{2}} \frac{(1-u)^{\alpha}-u^{\alpha}}{(u b+(1-u) a)^{2}}(1-u) d u\left|f^{\prime}(b)\right|^{q}\right)^{\frac{1}{q}} \\
+\left(\int_{\frac{1}{2}}^{1} \frac{u^{\alpha}-(1-u)^{\alpha}}{(u b+(1-u) a)^{2}} d u\right)^{1-\frac{1}{q}}\left(\int_{\frac{1}{2}}^{1} \frac{u^{\alpha}-(1-u)^{\alpha}}{(u b+(1-u) a)^{2}} u d u\left|f^{\prime}(a)\right|^{q} \int_{\frac{1}{2}}^{1} \frac{u^{\alpha}-(1-u)^{\alpha}}{(u b+(1-u) a)^{2}}(1-u) d u\left|f^{\prime}(b)\right|^{q}\right)^{\frac{1}{q}}
\end{array}\right]
\end{aligned}
$$


Calculating following integrals by Lemma 1, we have

$$
\begin{aligned}
& \int_{0}^{\frac{1}{2}} \frac{(1-u)^{\alpha}-u^{\alpha}}{(u b+(1-u) a)^{2}} d u \leq \int_{0}^{\frac{1}{2}} \frac{(1-2 u)^{\alpha}}{(u b+(1-u) a)^{2}} d u=\frac{1}{2} \int_{0}^{1} \frac{(1-u)^{\alpha}}{\left(\frac{u}{2} b+\left(1-\frac{u}{2}\right) a\right)^{2}} d u \\
& =2(a+b)^{-2} \int_{0}^{1} v^{\alpha}\left(1-v\left(\frac{b-a}{b+a}\right)\right)^{-2} d v \\
& =\frac{2(a+b)^{-2}}{(\alpha+1)}{ }_{2} F_{1}\left(2, \alpha+1 ; \alpha+2 ; \frac{b-a}{b+a}\right)=C_{3}(\alpha) \text {, } \\
& \int_{0}^{\frac{1}{2}} \frac{(1-u)^{\alpha}-u^{\alpha}}{(u b+(1-u) a)^{2}} u d u \leq \int_{0}^{\frac{1}{2}} \frac{(1-2 u)^{\alpha}}{(u b+(1-u) a)^{2}} u d u=\frac{1}{4} \int_{0}^{1} \frac{u(1-u)^{\alpha}}{\left(\frac{u}{2} b+\left(1-\frac{u}{2}\right) a\right)^{2}} d u \\
& =(a+b)^{-2} \int_{0}^{1}(1-v) v^{\alpha}\left(1-v\left(\frac{b-a}{b+a}\right)\right)^{-2} d u \\
& =\frac{(a+b)^{-2}}{(\alpha+1)(\alpha+2)}{ }_{2} F_{1}\left(2, \alpha+1 ; \alpha+3 ; \frac{b-a}{b+a}\right)=C_{4}(\alpha) \text {, } \\
& \int_{0}^{\frac{1}{2}} \frac{(1-u)^{\alpha}-u^{\alpha}}{(u b+(1-u) a)^{2}}(1-u) d u \leq C_{3}(\alpha)-C_{4}(\alpha)=C_{5}(\alpha) \\
& \int_{\frac{1}{2}}^{1} \frac{u^{\alpha}-(1-u)^{\alpha}}{(u b+(1-u) a)^{2}} d u=\int_{0}^{1} \frac{u^{\alpha}-(1-u)^{\alpha}}{(u b+(1-u) a)^{2}} d u+\int_{0}^{\frac{1}{2}} \frac{(1-u)^{\alpha}-u^{\alpha}}{(u b+(1-u) a)^{2}} d u \\
& \leq\left[\begin{array}{c}
\frac{b^{-2}}{(\alpha+1)}{ }_{2} F_{1}\left(2,1 ; \alpha+2 ; 1-\frac{a}{b}\right) \\
-\frac{b^{-2}}{(\alpha+1)}{ }_{2} F_{1}\left(2, \alpha+1 ; \alpha+2 ; 1-\frac{a}{b}\right)+C_{3}(\alpha)
\end{array}\right]=C_{6}(\alpha), \\
& \int_{\frac{1}{2}}^{1} \frac{u^{\alpha}-(1-u)^{\alpha}}{(u b+(1-u) a)^{2}} u d u=\int_{0}^{1} \frac{u^{\alpha}-(1-u)^{\alpha}}{(u b+(1-u) a)^{2}} u d u+\int_{0}^{\frac{1}{2}} \frac{(1-u)^{\alpha}-u^{\alpha}}{(u b+(1-u) a)^{2}} u d u \\
& \leq\left[\begin{array}{c}
\frac{b^{-2}}{(\alpha+2)}{ }_{2} F_{1}\left(2,1 ; \alpha+3 ; 1-\frac{a}{b}\right) \\
-\frac{b^{-2}}{(\alpha+1)(\alpha+2)}{ }_{2} F_{1}\left(2, \alpha+1 ; \alpha+3 ; 1-\frac{a}{b}\right)+C_{4}(\alpha)
\end{array}\right]=C_{7}(\alpha), \\
& \int_{\frac{1}{2}}^{1} \frac{u^{\alpha}-(1-u)^{\alpha}}{(u b+(1-u) a)^{2}}(1-u) d u \leq C_{6}(\alpha)-C_{7}(\alpha)=C_{8}(\alpha) .
\end{aligned}
$$

If we use $(27-32)$ in $(26)$, we have (25). This completes the proof.

Corollary 2. In Theorem 7, one has the following.

(1) If one takes $\alpha=1$, one has the following Hermite-Hadamard-Fejér inequality for harmonically convex functions which is related the right-hand side of (6):

$$
\begin{aligned}
& \left|\frac{f(a)+f(b)}{2} \int_{a}^{b} \frac{g(x)}{x^{2}} d x-\int_{a}^{b} \frac{f(x) g(x)}{x^{2}} d x\right| \leq \frac{\|g\|_{\infty}(b-a)^{2}}{2} \\
& \times\left[C_{3}^{1-\frac{1}{q}}(1)\left[\left(\begin{array}{c}
C_{4}(1)\left|f^{\prime}(a)\right|^{q} \\
+C_{5}(1)\left|f^{\prime}(b)\right|^{q}
\end{array}\right)\right]^{\frac{1}{q}}+C_{6}^{1-\frac{1}{q}}(1)\left[\left(\begin{array}{c}
C_{7}(1)\left|f^{\prime}(a)\right|^{q} \\
+C_{8}(1)\left|f^{\prime}(b)\right|^{q}
\end{array}\right)\right]^{\frac{1}{q}}\right],
\end{aligned}
$$


(2) If one takes $g(x)=1$, one has the following Hermite-Hadamard type inequality for harmonically convex function in fractional integral forms which is related the right-hand side of (5):

$$
\begin{aligned}
& \left|\frac{f(a)+f(b)}{2}-\frac{\Gamma(\alpha+1)}{2}\left(\frac{a b}{b-a}\right)^{\alpha}\left\{J_{1 / a-}^{\alpha}(f \circ h)(1 / b)+J_{1 / b+}^{\alpha}(f \circ h)(1 / a)\right\}\right| \leq \frac{a b(b-a)}{2} \\
& \times\left[C_{3}^{1-\frac{1}{q}}(\alpha)\left[\left(\begin{array}{c}
C_{4}(\alpha)\left|f^{\prime}(a)\right|^{q} \\
+C_{5}(\alpha)\left|f^{\prime}(b)\right|^{q}
\end{array}\right)\right]^{\frac{1}{q}}+C_{6}^{1-\frac{1}{q}}(\alpha)\left[\left(\begin{array}{c}
C_{7}(\alpha)\left|f^{\prime}(a)\right|^{q} \\
+C_{8}(\alpha)\left|f^{\prime}(b)\right|^{q}
\end{array}\right)\right]^{\frac{1}{q}}\right],
\end{aligned}
$$

(3) If one takes $\alpha=1$ and $g(x)=1$, one has the following Hermite-Hadamard type inequality for harmonically convex function which is related the right-hand side of (4):

$$
\begin{aligned}
& \left|\frac{f(a)+f(b)}{2}-\frac{a b}{b-a} \int_{a}^{b} \frac{f(x)}{x^{2}} d x\right| \leq \frac{a b(b-a)}{2} \\
& \times\left[C_{3}^{1-\frac{1}{q}}(1)\left[\left(\begin{array}{c}
C_{4}(1)\left|f^{\prime}(a)\right|^{q} \\
+C_{5}(1)\left|f^{\prime}(b)\right|^{q}
\end{array}\right)\right]^{\frac{1}{q}}+C_{6}^{1-\frac{1}{q}}(1)\left[\left(\begin{array}{c}
C_{7}(1)\left|f^{\prime}(a)\right|^{q} \\
+C_{8}(1)\left|f^{\prime}(b)\right|^{q}
\end{array}\right)\right]^{\frac{1}{q}}\right] .
\end{aligned}
$$

We can state another inequality for $q>1$ as follows.

Theorem 8. Let $f: I \subset(0, \infty) \rightarrow \mathbb{R}$ be a differentiable function on $I^{\circ}$ such that $f^{\prime} \in L[a, b]$, where $a, b \in I$ and $a<b$. If $\left|f^{\prime}\right|^{q}, q>1$, is harmonically convex on $[a, b], g:[a, b] \rightarrow \mathbb{R}$ is continuous and harmonically symmetric with respect to $2 a b / a+b$, then the following inequality for fractional integrals holds:

$$
\begin{aligned}
& \left|\frac{f(a)+f(b)}{2}\left[J_{1 / b+}^{\alpha}(g \circ h)(1 / a)+J_{1 / a-}^{\alpha}(g \circ h)(1 / b)\right]-\left[J_{1 / b+}^{\alpha}(f g \circ h)(1 / a)+J_{1 / a-}^{\alpha}(f g \circ h)(1 / b)\right]\right| \\
& \leq \frac{\|g\|_{\infty} a b(b-a)}{\Gamma(\alpha+1)}\left(\frac{b-a}{a b}\right)^{\alpha}\left[C_{9}^{\frac{1}{p}}(\alpha)\left[\frac{\left|f^{\prime}(a)\right|^{q}+3\left|f^{\prime}(b)\right|^{q}}{8}\right]^{\frac{1}{q}}+C_{10}^{\frac{1}{p}}(\alpha)\left[\frac{3\left|f^{\prime}(a)\right|^{q}+\left|f^{\prime}(b)\right|^{q}}{8}\right]^{\frac{1}{q}}\right]
\end{aligned}
$$

where

$$
\begin{aligned}
C_{9}(\alpha) & =\left(\frac{a+b}{2}\right)^{-2 p} \frac{1}{2(\alpha p+1)}{ }_{2} F_{1}\left(2 p, \alpha p+1 ; \alpha p+2 ; \frac{b-a}{b+a}\right), \\
C_{10}(\alpha) & =b^{-2 p} \frac{1}{2(\alpha p+1)}{ }_{2} F_{1}\left(2 p, 1 ; \alpha p+2 ; \frac{1}{2}\left(1-\frac{a}{b}\right)\right),
\end{aligned}
$$

with $0<\alpha \leq 1, h(x)=1 / x, x \in\left[\frac{1}{b}, \frac{1}{a}\right]$ and $1 / p+1 / q=1$.

Proof. Using (18), Hölder's inequality and the harmonically convexity of $\left|f^{\prime}\right|^{q}$, we have

$$
\begin{aligned}
& \left|\frac{f(a)+f(b)}{2}\left[J_{1 / b+}^{\alpha}(g \circ h)(1 / a)+J_{1 / a-}^{\alpha}(g \circ h)(1 / b)\right]-\left[J_{1 / b+}^{\alpha}(f g \circ h)(1 / a)+J_{1 / a-}^{\alpha}(f g \circ h)(1 / b)\right]\right| \\
& \leq \frac{\|g\|_{\infty} a b(b-a)}{\Gamma(\alpha+1)}\left(\frac{b-a}{a b}\right)^{\alpha}\left[\int_{0}^{\frac{1}{2}} \frac{(1-u)^{\alpha}-u^{\alpha}}{(u b+(1-u) a)^{2}}\left|f^{\prime}\left(\frac{a b}{u b+(1-u) a}\right)\right| d u\right. \\
& \left.+\int_{\frac{1}{2}}^{1} \frac{u^{\alpha}-(1-u)^{\alpha}}{(u b+(1-u) a)^{2}}\left|f^{\prime}\left(\frac{a b}{u b+(1-u) a}\right)\right| d u\right]
\end{aligned}
$$




$$
\begin{aligned}
& \leq \frac{\|g\|_{\infty} a b(b-a)}{\Gamma(\alpha+1)}\left(\frac{b-a}{a b}\right)^{\alpha}\left[\left(\int_{0}^{\frac{1}{2}} \frac{\left[(1-u)^{\alpha}-u^{\alpha}\right]^{p}}{(u b+(1-u) a)^{2 p}} d u\right)^{\frac{1}{p}}\left(\int_{0}^{\frac{1}{2}}\left|f^{\prime}\left(\frac{a b}{u b+(1-u) a}\right)\right|^{q} d u\right)^{\frac{1}{q}}\right. \\
& \left.+\left(\int_{\frac{1}{2}}^{1} \frac{\left[u^{\alpha}-(1-u)^{\alpha}\right]^{p}}{(u b+(1-u) a)^{2 p}} d u\right)^{\frac{1}{p}}\left(\int_{\frac{1}{2}}^{1}\left|f^{\prime}\left(\frac{a b}{u b+(1-u) a}\right)\right|^{q} d u\right)^{\frac{1}{q}}\right] \\
& \leq \frac{\|g\|_{\infty} a b(b-a)}{\Gamma(\alpha+1)}\left(\frac{b-a}{a b}\right)^{\alpha}\left[\left(\int_{0}^{\frac{1}{2}} \frac{\left[(1-u)^{\alpha}-u^{\alpha}\right]^{p}}{(u b+(1-u) a)^{2 p}} d u\right)^{\frac{1}{p}}\left(\int_{0}^{\frac{1}{2}} u\left|f^{\prime}(a)\right|^{q}+(1-u)\left|f^{\prime}(b)\right|^{q} d u\right)^{\frac{1}{q}}\right. \\
& \left.+\left(\int_{\frac{1}{2}}^{1} \frac{\left[u^{\alpha}-(1-u)^{\alpha}\right]^{p}}{(u b+(1-u) a)^{2 p}} d u\right)^{\frac{1}{p}}\left(\int_{\frac{1}{2}}^{1} u\left|f^{\prime}(a)\right|^{q}+(1-u)\left|f^{\prime}(b)\right|^{q} d u\right)^{\frac{1}{q}}\right] \\
& \leq \frac{\|g\|_{\infty} a b(b-a)}{2 \Gamma(\alpha+1)}\left(\frac{b-a}{a b}\right)^{\alpha} \times\left[\left(\int_{0}^{\frac{1}{2}} \frac{\left[(1-u)^{\alpha}-u^{\alpha}\right]^{p}}{(u b+(1-u) a)^{2 p}} d u\right)^{\frac{1}{p}}\left[\frac{\left|f^{\prime}(a)\right|^{q}+3\left|f^{\prime}(b)\right|^{q}}{8}\right]^{\frac{1}{q}}\right. \\
& +\left(\int_{\frac{1}{2}}^{1} \frac{\left[u^{\alpha}-(1-u)^{\alpha}\right]^{p}}{(u b+(1-u) a)^{2 p}} d u\right)^{\frac{1}{p}}\left[\frac{\left.3\left|f^{\prime}(a)\right|^{q}+\left|f^{\prime}(b)\right|^{q}\right]^{\frac{1}{q}}}{8}\right]
\end{aligned}
$$

Calculating following integrals by Lemma 1, we have

$$
\begin{aligned}
\int_{0}^{\frac{1}{2}} \frac{\left[(1-u)^{\alpha}-u^{\alpha}\right]^{p}}{(u b+(1-u) a)^{2 p}} d u & \leq \int_{0}^{\frac{1}{2}} \frac{(1-2 u)^{\alpha p}}{(u b+(1-u) a)^{2 p}} d u=\frac{1}{2} \int_{0}^{1} \frac{(1-u)^{\alpha p}}{\left(\frac{u}{2} b+\left(1-\frac{u}{2}\right) a\right)^{2 p}} d u \\
& =\frac{1}{2} \int_{0}^{1} v^{\alpha p}\left(\frac{a+b}{2}\right)^{-2 p}\left[1-v\left(\frac{b-a}{b+a}\right)\right]^{-2 p} d v \\
& =\left(\frac{a+b}{2}\right)^{-2 p} \frac{1}{2(\alpha p+1)}{ }_{2} F_{1}\left(2 p, \alpha p+1 ; \alpha p+2 ; \frac{b-a}{b+a}\right)=C_{9}(\alpha)
\end{aligned}
$$

and similarly

$$
\begin{aligned}
\int_{\frac{1}{2}}^{1} \frac{\left[u^{\alpha}-(1-u)^{\alpha}\right]^{p}}{(u b+(1-u) a)^{2 p}} d u & \leq \int_{\frac{1}{2}}^{1} \frac{(2 u-1)^{\alpha p}}{(u b+(1-u) a)^{2 p}} d u=\int_{0}^{\frac{1}{2}} \frac{(1-2 u)^{\alpha p}}{(u a+(1-u) b)^{2 p}} d u \\
& =\frac{1}{2} \int_{0}^{1} \frac{(1-v)^{\alpha p}}{\left(\frac{v}{2} a+\left(1-\frac{v}{2}\right) b\right)^{2 p}} d v=\frac{1}{2} \int_{0}^{1}(1-v)^{\alpha p} b^{-2 p}\left(1-\frac{v}{2}\left(1-\frac{a}{b}\right)\right)^{-2 p} d v \\
& =b^{-2 p} \frac{1}{2(\alpha p+1)}{ }_{2} F_{1}\left(2 p, 1 ; \alpha p+2 ; \frac{1}{2}\left(1-\frac{a}{b}\right)\right)=C_{10}(\alpha) .
\end{aligned}
$$

If we use (35) and (36) in (34), we have (33).This completes the proof.

Corollary 3. In Theorem 8, one has the following.

(1) If one takes $\alpha=1$, one has the following Hermite-Hadamard-Fejér inequality for harmonically convex functions which is related the right-hand side of (6):

$$
\left|\frac{f(a)+f(b)}{2} \int_{a}^{b} \frac{g(x)}{x^{2}} d x-\int_{a}^{b} \frac{f(x) g(x)}{x^{2}} d x\right| \leq \frac{\|g\|_{\infty}(b-a)^{2}}{2}\left[\begin{array}{c}
C_{9}^{\frac{1}{p}}(1)\left[\frac{\left|f^{\prime}(a)\right|^{q}+3\left|f^{\prime}(b)\right|^{q}}{8}\right]^{\frac{1}{q}} \\
+C_{10}^{\frac{1}{p}}(1)\left[\frac{3\left|f^{\prime}(a)\right|^{q}+\left|f^{\prime}(b)\right|^{q}}{8}\right]^{\frac{1}{q}}
\end{array}\right],
$$


(2) If one takes $g(x)=1$, one has the following Hermite-Hadamard type inequality for harmonically convex function in fractional integral forms which is related the right-hand side of (5):

$$
\begin{aligned}
& \left|\frac{f(a)+f(b)}{2}-\frac{\Gamma(\alpha+1)}{2}\left(\frac{a b}{b-a}\right)^{\alpha}\left\{J_{1 / a-}^{\alpha}(f \circ h)(1 / b)+J_{1 / b+}^{\alpha}(f \circ h)(1 / a)\right\}\right| \leq \frac{a b(b-a)}{2} \\
& \times\left[C_{9}^{\frac{1}{p}}(\alpha)\left[\frac{\left|f^{\prime}(a)\right|^{q}+3\left|f^{\prime}(b)\right|^{q}}{8}\right]^{\frac{1}{q}}+C_{10}^{\frac{1}{p}}(\alpha)\left[\frac{3\left|f^{\prime}(a)\right|^{q}+\left|f^{\prime}(b)\right|^{q}}{8}\right]^{\frac{1}{q}}\right],
\end{aligned}
$$

(3) If one takes $\alpha=1$ and $g(x)=1$,one has the following Hermite-Hadamard type inequality for harmonically convex function which is related the right-hand side of (4):

$$
\left|\frac{f(a)+f(b)}{2}-\frac{a b}{b-a} \int_{a}^{b} \frac{f(x)}{x^{2}} d x\right| \leq \frac{a b(b-a)}{2}\left[\begin{array}{c}
C_{9}^{\frac{1}{p}}(1)\left[\frac{\left|f^{\prime}(a)\right|^{q}+3\left|f^{\prime}(b)\right|^{q}}{8}\right]^{\frac{1}{q}} \\
+C_{10}^{\frac{1}{p}}(1)\left[\frac{3\left|f^{\prime}(a)\right|^{q}+\left|f^{\prime}(b)\right|^{q}}{8}\right]^{\frac{1}{q}}
\end{array}\right]
$$

\section{Conclusions}

In this paper, Hermite-Hadamard-Fejer type inequalities for harmonically convex functions in fractional integral forms are given. Also, an integral identity and some trapezoidal Hermite-Hadamard-Fejer type integral inequalities for harmonically convex functions in fractional integral forms are obtained.

\section{Competing Interests}

The authors declare that they have no competing interests.

\section{Authors' Contributions}

All authors have contributed to all parts of the article. All authors read and approved the final manuscript.

\section{References}

[1] M. Bombardelli and S. Varošanec, Properties of $h$-convex functions related to the Hermite Hadamard Fejér inequalities, Computers and Mathematics with Applications 58 (2009), 18691877.

[2] F. Chen and S. Wu, Fejér and Hermite-Hadamard type inqequalities for harmonically convex functions, Jurnal of applied Mathematics, volume 2014, article id:386806.

[3] Z. Dahmani, On Minkowski and Hermite-Hadamard integral inequalities via fractional integration, Ann. Funct. Anal. 1(1) (2010), 51-58.

[4] L. Fejér, Uberdie Fourierreihen, II, Math. Naturwise. Anz Ungar. Akad., Wiss, 24 (1906), 369-390, (in Hungarian).

[5] J. Hadamard, Étude sur les propriétés des fonctions entières et en particulier d'une fonction considérée par Riemann, J. Math. Pures Appl., 58 (1893), 171-215.

[6] İ. İşcan, New estimates on generalization of some integral inequalities for $s$-convex functions and their applications, Int. J. Pure Appl. Math., 86(4) (2013), 727-746.

[7] İ. İşcan, Some new general integral inequalities for $h$-convex and $h$-concave functions, Adv. Pure Appl. Math. 5(1) (2014), 21-29 . doi: 10.1515/apam-2013-0029. 
[8] İ. İşcan, Generalization of different type integral inequalitiesfor $s$-convex functions via fractional integrals, Applicable Analysis, 2013. doi: 10.1080/00036811.2013.851785.

[9] İ. İşcan, On generalization of different type integral inequalities for $s$-convex functions via fractional integrals, Mathematical Sciences and Applications E-Notes, 2(1) (2014), 55-67.

[10] İ. İşcan, S. Wu, Hermite-Hadamard type inequalities for harmonically convex functions via fractional integrals, Appl. Math. Comput., 238 (2014) 237-244.

[11] İ. İşcan, Hermite-Hadamard type inequalities for harmonically convex functions, Hacet. J. Math. Stat., 43 (6) (2014), $935-942$

[12] A. A. Kilbas, H. M. Srivastava, J. J. Trujillo, Theory and applications of fractional differential equations. Elsevier, Amsterdam (2006).

[13] M. A. Latif, S. S. Dragomir and E. Momoniat, Some Fejér type inequalities for harmonically-convex functions with applications to special means, http://rgmia.org/papers/v18/v18a24.pdf.

[14] A. P. Prudnikov, Y. A. Brychkov, O. J. Marichev, Integral and series, Elementary Functions, vol. 1, Nauka, Moscow, 1981.

[15] M.Z. Sarıkaya, On new Hermite Hadamard Fejér type integral inequalities, Stud. Univ. Babeş-Bolyai Math. 57(3) (2012), 377-386.

[16] M.Z. Sarıkaya, E. Set, H. Yaldız and N. Başak, Hermite-Hadamard's inequalities for fractional integrals and related fractional inequalities, Mathematical and Computer Modelling, 57(9) (2013), 2403-2407.

[17] K.-L. Tseng, G.-S. Yang and K.-C. Hsu, Some inequalities for differentiable mappings and applications to Fejér inequality and weighted trapezoidal formula, Taiwanese journal of Mathematics, 15(4) (2011), 1737-1747.

[18] J. Wang, X. Li, M. Fečkan and Y. Zhou, Hermite-Hadamard-type inequalities for Riemann-Liouville fractional integrals via two kinds of convexity, Appl. Anal., 92(11) (2012), 2241-2253. doi:10.1080/00036811.2012.727986

[19] J. Wang, C. Zhu and Y. Zhou, New generalized Hermite-Hadamard type inequalities and applications to special means, J. Inequal. Appl., 2013(325) (2013), 15 pages. 